EPJ Web of Conferences 41, 11009 (2013)

DOI: $10.1051 /$ epjconf/20134111009

C) Owned by the authors, published by EDP Sciences, 2013

\title{
Pulse-shaping-based two-photon FRET microscopy
}

\author{
M. H. Brenner ${ }^{1}$, D. Cai ${ }^{2}$, S. W. Straight ${ }^{3}$, J. A. Swanson ${ }^{3}$, and J. P. Ogilvie ${ }^{2}$ \\ ${ }^{1}$ Applied Physics Program, University of Michigan, 450 Church Street, Ann Arbor, MI, USA 48109 \\ ${ }^{2}$ Department of Physics and Biophysics, University of Michigan, 450 Church Street, Ann Arbor, MI, \\ USA 48109 \\ ${ }^{3}$ Department of Microbiology and Immunology, University of Michigan Medical School, 1150 West \\ Medical Center Drive, Ann Arbor, MI, USA 48109
}

\begin{abstract}
We present quantitative pulse-shaping-based two-photon fluorescence resonance energy transfer microscopy. We tailor the spectral phase of the excitation pulses to achieve selective excitation of donor and acceptor, demonstrating the method in live cells.
\end{abstract}

Fluorescence Resonance Energy Transfer (FRET) microscopy has been used extensively in biophysics for studying phenomena ranging from protein folding pathways to cellular signalling [1]. A major impediment to obtaining quantitative FRET measurements is the fact that it is often difficult to avoid direct excitation of the acceptor, which produces fluorescence that can be misinterpreted as FRET [2]. In one-photon FRET studies, FRET stoichiometry methods have been developed to resolve this problem, providing quantitative FRET measurements [2]. This approach requires selective excitation of donor and acceptor, and acquisition of separate images from donor and acceptor fluorescence channels. Analogous two-photon FRET stoichiometry has not yet been performed due to the challenges of performing rapid, multicolor two-photon imaging. Typical twophoton fluorescence microscopes utilize $\sim 100$ fs titanium sapphire oscillators as the excitation source. With such a source, different fluorophores are excited by tuning the central wavelength. Since tuning is typically a slow process ( $\sim$ seconds), rapid multicolor imaging is difficult unless multiple laser sources are used. An attractive alternative is to use broadband lasers, with bandwidths of $>100 \mathrm{~nm}$. While such bandwidths enable the simultaneous excitation of multiple fluorophores, the lack of selectivity for separate excitation of donor and acceptor species complicates the implementation of FRET stoichiometry. Here we use pulse-shaping as a method to achieve selective donor and acceptor excitation, resolving the problem of spectral overlap that will enable two-photon FRET stoichiometry.

Pulse-shaping methods have been used previously in microscopy applications for selective excitation [3-5] and imaging of a FRET-based calcium indicator [6]. Here we use two different phase masks designed to selectively excite donor (mCerulean) and acceptor (mCherry) fluorescent proteins in live COS-7 cells. Rapid switching between phase-masks ( $\sim 10 \mathrm{~ms})$ enables rapid collection of the images required for FRET stoichiometry. The phase masks were tailored using binary phase shaping [7] to excite the fluorophores in the regions of their two-photon absorption spectra that would lead to optimal contrast. As a result, mCherry was excited via an $\mathrm{S}_{0}-\mathrm{S}_{\mathrm{n}}$ transition using a shaped pulse centered at shorter wavelengths than those used to excite mCerulean. A transmissive $4 \mathrm{f}$ pulse shaper setup containing a CRi 640 pixel, phase-only spatial light modulator (SLM) was used to shape input

This is an Open Access article distributed under the terms of the Creative Commons Attribution License 2.0, which permits unrestricted use, distribution, and reproduction in any medium, provided the original work is properly cited. 
pulses from a $75 \mathrm{MHz}$ titanium:sapphire oscillator (Femtolasers Synergy) with 110nm of bandwidth centered at $780 \mathrm{~nm}$. Approximately $5.5 \mathrm{~mW}$ was used for excitation. Images were acquired with a Prairie Technologies-modified upright Olympus microscope and analyzed in Matlab. Two sets of live Cos-7 cells were imaged. The "unlinked" sample cells expressed both free mCerulean and mCherry such that there was minimal likelihood of FRET between them. The "linked" sample cells expressed a construct of linked mCerulean and mCherry with a measured FRET efficiency of $22.19 \%$ (determined using fluorescence lifetime imaging). The cells were given 24 hours for transient expression of the proteins, and they were kept at $37^{\circ} \mathrm{C}$ throughout imaging.

When cells in the unlinked sample were excited by an unshaped, transform-limited (TL) pulse, both the mCerulean and mCherry proteins fluoresced, with little discrimination between them. Applying the shaped pulses, however, led to enhanced contrast. Under excitation designed to preferentially excite mCerulean, blue contrast was $73 \%$, where contrast is defined as (mCerulean fluorescence - mCherry fluorescence)/mCerulean fluorescence. However, when the pulse shaped to preferentially excite mCherry was used, red contrast of $49 \%$ was achieved, where red contrast is defined as (mCherry fluorescence - mCerulean fluorescence)/mCherry fluorescence. Before performing calculations, these images were normalized to their TL counterparts to correct for differences in transfection efficiency and brightness of the fluorophores. These results indicate that we are able to selectively excite the donor and acceptor fluorophores in live cells.

For the linked condition, the expected results are different due to the effects of FRET. When the donor is excited, it will transfer some of its energy to the acceptor, resulting in a decrease in donor fluorescence and an increase in acceptor fluorescence. Consequently, when a pulse shaped to preferentially excite mCerulean is used, we expect to see a decrease in blue contrast due to energy being transferred to the acceptor. When a pulse shaped to preferentially excite mCherry is used, we expect to see an increase in red contrast. This is because although we are primarily exciting the acceptor, due to spectral overlap we are also weakly exciting the donor. Once excited, the donor can still transfer energy to the acceptor, leading to an increase in fluorescence signal from the acceptor.

Figure 1 shows the results from the FRET condition. mCherry signal is shown on the left and mCerulean signal is shown on the right. The top row shows that when the unshaped, TL pulse is used, both fluorophores are excited. Excitation with the pulse shaped to preferentially excite mCerulean is shown in the middle row. More blue fluorescence than red is observed, but the blue contrast has decreased as expected, from $73 \%$ in the No FRET condition to $53 \%$. The bottom row shows images of the cell excited by a pulse tuned to excite mCherry. As expected, the red contrast has increased from $49 \%$ in the No FRET condition, to $69 \%$, consistent with the detection of FRET. Similar results were seen for multiple cells and applications of the pulse shapes.

This work demonstrates selective two-photon excitation of fluorescent proteins in live cells, and detection of FRET between them, using ultrafast laser pulse-shaping. Compared to other twophoton FRET microscopy methods, this technique has advantages such as decreased signal from accidental direct excitation and improved temporal resolution. This pulse-shaping approach will enable future applications of two-photon FRET stoichiometry. 


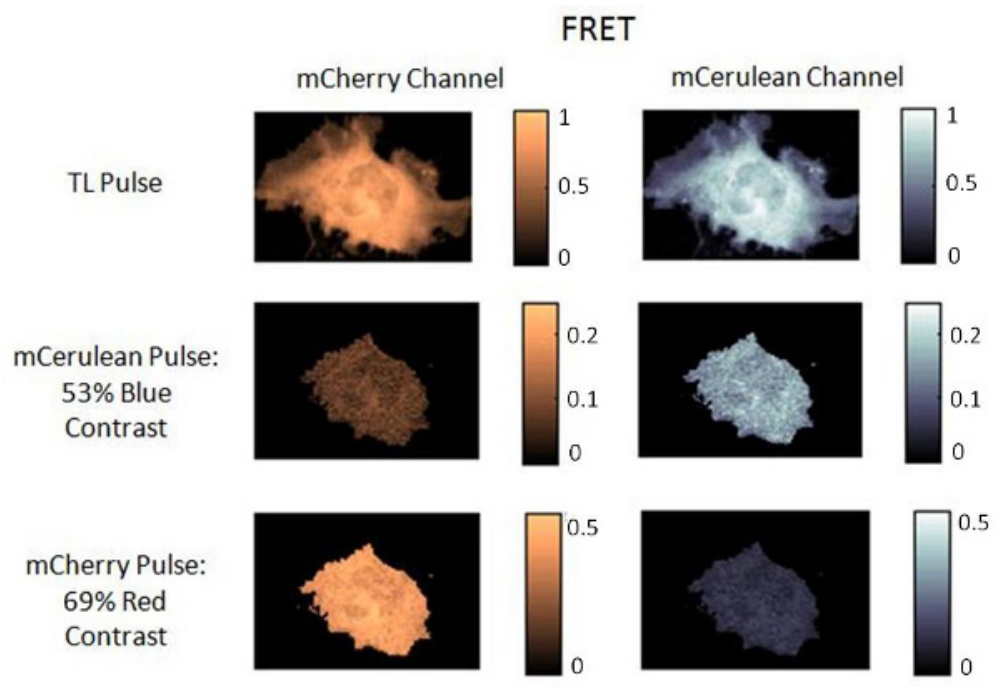

Fig. 1. Live Cos7 cells expressing linked mCerulean and mCherry. The top row shows the cell excited by the unshaped, TL pulse; both fluorophores are excited. The middle row shows excitation by a pulse tuned to preferentially excite mCerulean; blue contrast (53\%) is less than that seen for the No FRET condition (73\%). The bottom row shows excitation by a pulse shaped to preferentially excite mCherry; red contrast (69\%) is greater than that seen for the No FRET condition (49\%). The contrast changes are due to transferred energy appearing as decreases in blue fluorescence and increases in red fluorescence.

\section{Acknowledgements}

We gratefully acknowledge support from the National Institutes of Health (grant \# 1-R21-EB012686-01-A1). M.H. Brenner was supported by an NIH Molecular Biophysics Training Grant and a University of Michigan Rackham Merit Fellowship.

\section{References}

[1] P. R. Selvin, "Fluorescence resonance energy transfer," Biochemical Spectroscopy, 246, 300 (1995)

[2] A. Hoppe, K. Christensen, and J. A. Swanson, Biophysical Journal, 83, 3652, (2002)

[3] R. S. Pillai, C. Boudoux, G. Labroille, N. Olivier, I. Veilleux, E. Farge, M. Joffre, and E. Beaurepaire, Optics Express, 17, 12741, (2009)

[4] J. P. Ogilvie, D. Debarre, X. Solinas, J. L. Martin, E. Beaurepaire, and M. Joffre, Optics Express, 14, 759, (2006)

[5] P. Xi, Y. Andegeko, L. R. Weisel, V. V. Lozovoy, and M. Dantus, Optics Communications, 281, $1841,(2008)$

[6] K. Isobe, A. Suda, M. Tanaka, F. Kannari, H. Kawano, H. Mizuno, A. Miyawaki, and K.

Midorikawa, Optics Express, 17, 13737, (2009)

[7] M. Comstock, V. V. Lozovoy, I. Pastirk, and M. Dantus, Optics Express, 12, 1061, (2004) 